\title{
Pump-Probe Experiments in the Femtosecond Regime, Combining First and Third Harmonics of SASE FEL Radiation
}

\author{
J. Feldhaus, T. Möller, E. L. Saldin, E. A. Schneidmiller \\ Deutsches Elektronen-Synchrotron DESY, Hamburg \\ M. V. Yurkov \\ Joint Institute for Nuclear Research, Dubna, Moscow Region, Russia
}




\title{
Pump-probe experiments in the femtosecond regime, combining first and third harmonics of SASE FEL radiation
}

\author{
J. Feldhaus $^{\text {a }}$, T. Möller ${ }^{\text {a }}$, E.L. Saldin ${ }^{\text {a }}$, E.A. Schneidmiller ${ }^{\text {a }}$, \\ and M.V. Yurkov ${ }^{b}$ \\ a Deutsches Elektronen-Synchrotron (DESY), Notkestrasse 85, D-22607 Hamburg, \\ Germany \\ b Joint Institute for Nuclear Research, Dubna, 141980 Moscow Region, Russia
}

\begin{abstract}
Two-color pump-probe experiments combining optical femtosecond lasers with short wavelength radiation from a free electron laser (FEL) are very attractive for sub-picosecond time-resolved stadies. Since the synchronization between the two light sources to an accuracy of 100 femtoseconds is not yet solved, it is proposed to derive both radiation pulses from the same electron bunch. In the present work we focus on the special case where pump and probe beams are generated by the same electron bunch in the same insertion device. Specifically we propose to combine GWlevel VUV FEL pulses between $150 \mathrm{~nm}$ and $90 \mathrm{~nm}$ wavelength and $10 \mathrm{MW}$-level third-harmonic radiation between $50 \mathrm{~nm}$ and $30 \mathrm{~nm}$. This scheme does not require any special synchronization nor additional FEL hardware components since the nonlinear third-harmonic generation occurs naturally in the planar FEL undulator. Reflection optics is used for beam splitting and tunable delay, the two harmonics are separated by using notch filters. The effect on the pulse duration is negligible.
\end{abstract}

Over the last thirty years synchrotron radiation has become a very powerful research tool applied in many different fields of science, in physics, chemestry and biology. The rapid progress was driven by the development of new, increasingly brilliant synchrotron radiation sources based on electron or positron storage rings. Recently a new era of synchrotron radiation research has begun with first user experiments on a free electron laser (FEL) based on self-amplified spontaneous emission (SASE). The results have been obtained at the TESLA Test Facility (TTF) at DESY (Hamburg) [1,2] using radiation pulses of $100 \pm$ $5 \mathrm{~nm}$ wavelength with $100 \mathrm{fs}$ pulse duration and peak power of approximately $1 \mathrm{GW}$ [3]. The peak brilliance of this SASE FEL is nine orders of magnitude higher than that of modern, third generation synchrotron light sources, and 
the light pulses are three orders of magnitude shorter. The average brilliance is orders of magnitude higher too, and the TTF FEL may thus be considered as the first 4 th generation VUV light source in the world.

SASE FELs hold great promise as a source of short wavelength radiation down to $0.1 \mathrm{~nm}$ for a wide range of applications, particularly for the study of fast dynamic processes on a sub-picosecond time scale. The standard technique for high resolution time resolved measurements is a pump-probe scheme in which a process is started by a short pulse of radiation (pump) and the evolution of the process is then observed (probed) at a different times after the start by means of a second short pulse of radiation, generally at another photon energy. For very high resolution studies with optical lasers in the pico- and femtosecond regime the two radiation pulses are always derived from the same laser to ensure perfect synchronization, and the time difference is adjusted by changing the path length through an optical delay line. Pump-probe experiments combining pulses from a FEL and an optical laser are more difficult for two reasons. First, the linear accelerator is normally operated with a pulsetiming which is unusual for optical lasers. Second, the geometrical distance between the electron gun of the accelerator and the FEL user experiments can be hundreds of meters or even a few kilometers (for X-ray FEL) making perfect synchronization almost impossible. Two types of photon-pump-photon probe experiments have been proposed for the SASE FEL at the TTF:

1) independent pump and probe light sources employing a powerful optical laser system synchronized with the FEL [4],

2) pump and probe beams that are generated by the same electron bunch but from two different insertion devices [5].

Independent pump and probe sources require synchronization to enable standard time-delay schemes, where the arrival time of the probe relative to the pump can be predetermined by using delay lines. The best synchronization between two sources achieved to date is of the order of a picoseconds [7]. Short wavelength SASE FELs can provide radiation pulses shorter than 100 femtoseconds, hence the synchronization should be better, possibly down to a few 10 femtoseconds. Even if the same optical laser used in the pump-probe experiment also generated the electron bunch producing the FEL radiation pulse, it is not clear how well the accelerator can be stabilized to keep the travel time of the electron bunches constant on a 100 fs-level or even better. Sub-picosecond synchronization certainly needs further research and development. On the other hand, if the two different frequencies were generated by the same electron bunch, the critical synchronization could be avoided and only stable optical delay would be required. This technique has been applied previously combining synchrotron radiation and VUV radiation from a storage ring FEL, although on a much longer time scale [6]. This method could be 
a very interesting alternative to the optical laser- SASE FEL approach, and it has the further advantage to make a wide frequency range accessible at very high average power not so easily available from conventional lasers. It should be possible to achieve a timing accuracy close to the duration of the radiation pulses (100 fs), allowing unprecedented insight into the dynamics of electronic exitations, chemical reactions and phase transitions of matter, from atoms, through organic and inorganic molecules and clusters, to surface, solids and plasmas.

In order to test this method it is proposed to exploit the ultrashort intense SASE radiation pulses from the TTF FEL and to start with a simple experiment combining pump and probe beams produced by the same electron bunch in the same insertion device. The analaysis shows that strong harmonic growth can be expected In SASE FELs. When a beam is strongly bunched in the sinusoidal ponderomotive potential formed by the undulator field and the radiation field of the fundamental frequency, the electron beam density spectrum develops reach harmonic contents. Coherent radiation at the odd harmonics can be generated in a planar undulator and significant power levels for the third harmonic can be reached before the FEL saturates [8]. Explicit calculations based on current SASE FEL projects show that the power of the transversely coherent third-harmonic radiation can approach $1 \%$ of the fundamental power level $[9,10]$. The high harmonic power levels indicate the possibility of combining them with the FEL fundamental frequency in the pump-probe experiment.

It is therefore proposed to make use of the GW-level VUV FEL pulse as a pump and $10 \mathrm{MW}$-level third-harmonic radiation from the same SASE FEL as a probe pulse or vice versa. Since the nonlinear harmonic generation occurs naturally in planar SASE FEL undulators, no special synchronization nor any additional FEL hardware components are required for this experiment. It is only necessary to develop components to select the 1st and 3rd harmonic pulses and combine them with a tunable delay.

A basic scheme of the proposed pump-probe facility is shown in Fig. 1. The general characteristics for a 1st harmonic wavelength of $150 \mathrm{~nm}$ are listed in the Table 1. For a test experiment in the range of 150 to $90 \mathrm{~nm}$ for the 1 st harmonic and 50 to $30 \mathrm{~nm}$ for the $3 \mathrm{rd}$ harmonic the simplest way to separate the two frequencies is to use notch filters, such as $\mathrm{MgF}_{2}$, In or Al. The specific design of a delay line with filters is shown in Fig. 2. A single FEL pulse passes beam splitter, generating two pulses travelling different paths. The two pulses are recombined on the sample with a time difference $\Delta t$ which can be varied by changing the relative mirror position. Using beam splitting techniques, the time delay can be precisely controlled, and the temporal resolution is only limited by the stability of the optics used to relative to the other. A resolution of some ten femtoseconds should be achievable without much effort. The $\mathrm{Al}$ 


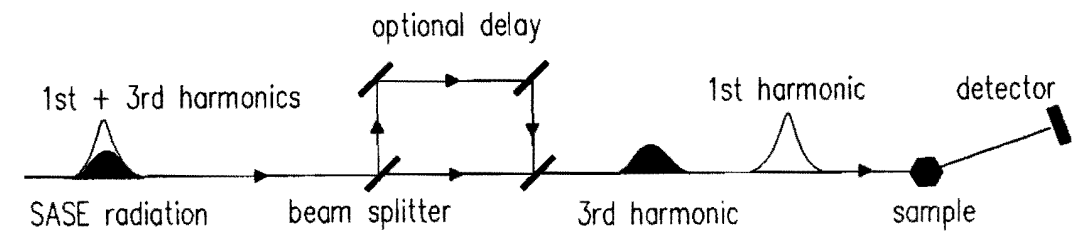

Fig. 1. Scheme for pump-probe experiments employing 1st SASE FEL harmonic radiation pulse as a pump and 3rd harmonic pulse as a probe or vice versa.

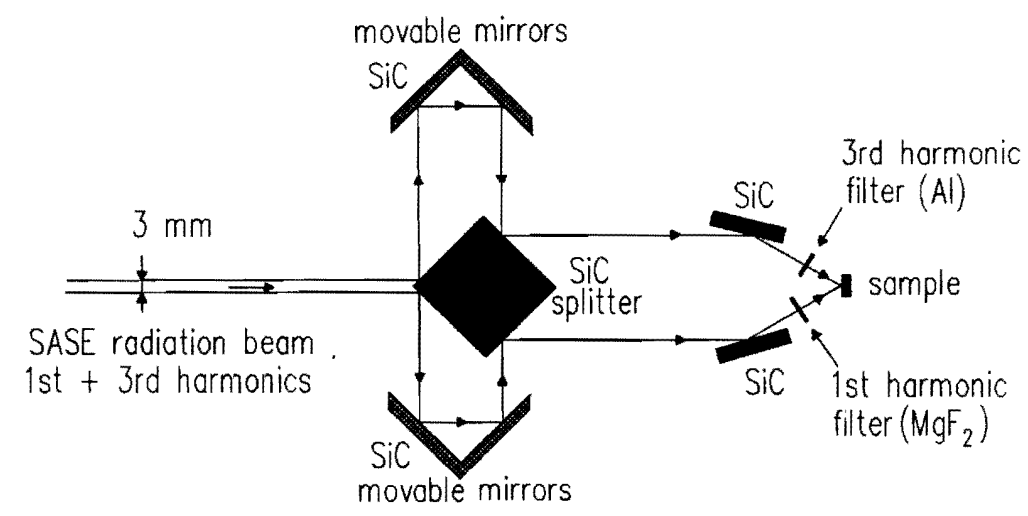

Fig. 2. Schematic setup of a delay line for pump-probe experiments. A SASE pulse passes a beam splitter, generating two pulses travelling different paths. After this, the two pulses impinge on the sample at the time difference which can be varied by changing the relative mirror position.

filter is useful in the $50-30 \mathrm{~nm}$ region. The transmission $T$ of the $100 \mathrm{~nm}$ thick Al filter increases from $63 \%$ to $72 \%$ for wavelengths between $50 \mathrm{~nm}$ and 30 $\mathrm{nm}$, while the fundamental is strongly suppressed $\left(T\right.$ varies from $1.6 \times 10^{-6}$ to $1.5 \times 10^{-3}$ for wavelengths between $150 \mathrm{~nm}$ and $90 \mathrm{~nm}$ ). The $\mathrm{SiC}$ mirrors are best suited for delay lines in this spectral range. The mirror reflectivity for s-polarized light at an angle of incidence of $45^{\circ}$ is about $60 \%$ and $45 \%$ for wavelengths of $150 \mathrm{~nm}$ and $50 \mathrm{~nm}$, respectively. Consequently, the transmission of the delay lines are about $14 \%$ and $4 \%$ for $150 \mathrm{~nm}$ and $50 \mathrm{~nm}$ respectively.

For other wavelengths, or for very high power levels, simple transmission filters may not be available, and a monochromator has to the used to select the two wavelengths. In this case one has to take care that the short pulse duration is preserved. In princilpe, it is possible to design time-compensated spectroscopic configurations with gratings by using at least two gratings in a substrative 
Table 1

Major parameters of VUV-EUV TTF SASE FEL pump-probe facility

\begin{tabular}{lc}
\hline Parameter & Value \\
\hline & \\
Wavelength of the 1st harmonic & $150 \mathrm{~nm}$ \\
bandwidth & $1 \%$ \\
SASE pulse duration & $100 \mathrm{fs}$ \\
number of photons at the FEL exit & \\
1st harmonic pulse & $10^{14}$ \\
3rd harmonic pulse & $10^{12}$ \\
number of pulses per train & 100 \\
repetition rate & $1 \mathrm{~Hz}$ \\
delay line transmission & \\
for 1st harmonic & $14 \%$ \\
for 3rd harmonic & $4 \%$ \\
\hline
\end{tabular}

fashion to compensate for the dispersion. This concept is well known in the study of ultrafast laser pulses, and has recently been extended to the EUV region [11].

Single harmonics can also be selected by using plane multilayer mirrors near normal incidence. The design of time-compensated monochromator without diffraction gratings has been presented in [12]. The monochromator is useful in the range 4-35 $\mathrm{nm}$ region, where multilayer mirrors have good performance. The pulse time duration is not altered up to a few femtoseconds, preserving the time resolution capability and the peak intensity.

\section{Acknowledgments}

We thank J.R. Schneider and D. Trines for interest in this work.

\section{References}

[1] J. Rossbach, Nucl. Instrum. and Methods A375(1996)269

[2] J. Andruszkow et al., Phys. Rev. Lett. 85(2000)3825 
[3] V. Ayvazayn et al., submitted to Phys. Rev. Lett.

[4] "Development of a pump-probe facility with sub-picisecond time resolution combining a high-power optical laser and a soft X-ray free electron laser": Joint DESY (Germany), Forshungszentrum Julich (Germany), Max-Born Institute Berlin (Germany), Dublin City University (Ireland), MAX-Lab/Lund Laser Centre (Sweden), and CNRS/LURE, orsay (France) Proposal. Available at DESY by request only

[5] B. Faatz et al., DESY-print 00-94(2000)

[6] L. Nahon et al., Nucl. Instrum. Methods Phys. Res. A 429, 489(1999)

[7] G. M. H. Knippels, M. J. van de Pol and A.F.G. van der Meer, Proceedings of the 20th International FEL Conference, Williamsburg, USA, 1998, Elsevier Science B.V., Amsterdam, 1999, p. II-97

[8] R. Bonifacio, L. De Salvo, and P. Pierini, Nucl. Instrum. Methods Phys. Res. A 293, 627(1990)

[9] H. P. Freund, S. G. Biedron, and S. V. Milton, Nucl. Instrum. Methods Phys. Res. A 445, 53(2000)

[10] Z. Huang and K. -J. Kim, Phys. Rev. E 62 7295(2000)

[11] P. Viloresi, Appl. Opt. 23, 293(1999)

[12] L. Poletto and G. Tondello, Journal of Opt. A 3 374(2001) 